EPJ Web of Conferences 40, 17005 (2013)

DOI: $10.1051 /$ epjconf/20134017005

(c) Owned by the authors, published by EDP Sciences, 2013

\title{
A soft magnetic material for power supply systems of high energy physics experiments
}

\author{
S. Baccaro ${ }^{1,5}$, P. Cova $^{2,4}$, N. Delmonte ${ }^{2,4}$, E. Ghisolfi ${ }^{3}$, and A. Lanza ${ }^{4}$ \\ ${ }^{1}$ UTT-MATT Casaccia Res. Centre, ENEA, via Anguillarese 301, I-00060 Santa Maria di Galeria (Rome), Italy \\ ${ }^{2}$ Dipartmento Ingegneria dell'Informazione, University of Parma, viale G.P. Usberti 181/A, I-43124 Parma, Italy \\ ${ }^{3}$ FN SpA Nuove Tecnologie e Servizi Avanzati, Strada per Crescentino 41, I-13040 Saluggia (VC), Italy \\ ${ }^{4}$ Istituto Nazionale di Fisica Nucleare - Sezione di Pavia, via A. Bassi, 6, I-27100 Pavia, Italy \\ ${ }^{5}$ Istituto Nazionale di Fisica Nucleare - Sezione di Roma, piazzale A. Moro, 2, I-00185 Rome, Italy
}

\begin{abstract}
Most of the high energy physics experiments require their detectors to be embedded in a high intensity magnetic field. In particular the biggest of them, ATLAS, running in the CERN Large Hadron Collider (LHC) particle accelerator, generates a field of $2 \mathrm{~T}$ by means of a gigantic toroidal magnet working in open air. Its future phase 2 upgrade plans to move the $\mathrm{DC} / \mathrm{DC}$ power supplies from the present positions on the external balconies directly on the detectors, where the field is of the order of $1 \mathrm{~T}$. This presentation describes the development of samples made of special magnetic material for inductor cores suitable to work in such an environment.

Starting from iron-silicon powders, at FN plant a plastic forming process, based on powder extrusion, injection moulding and sintering, was developed. To get the best compromise between the forming process requirements (good coupling among the metallic powder and the organic components to assure the right mouldability) and the debinding and sintering conditions, several mixtures (with different percentages and kind of organic additives) were experimented. A proper mould was designed and realized to get torous-shaped prototypes.

The preliminary results of the physical-microstructural characterization performed on the first prototypes made will be shown.
\end{abstract}

\section{Introduction}

The upgrades of the Large Hadron Collider (LHC) experiments at the Conseil Européen pour la Recherche Nucléaire (CERN) in Geneva, Switzerland, are planned for the beginning of the next decade. They require, in particular for the ATLAS experiment [1], a thorough redesign of the architecture of the power distribution system for many detectors. The requirements of new front-end electronics and the increase of the radiation background, about a factor of 5 above the present nominal values, are indeed unsuited with the current capability of the distribution systems in use. A possible solution involves the development of a distributed power supply network, with point of load converters deployed at the very heart of the detectors where electronic devices must face a highly hostile environment, in terms of very high background of both charged and neutral particles $[2,3]$ and for the presence of a non negligible magnetic field up to $100 \mathrm{mT}$, thus opening a severe tolerance issue for component selection and system design.

Our investigation proposes a new power supply distribution network, taking the ATLAS Muon spectrometer as the case study. Figure 1 [4] represents a

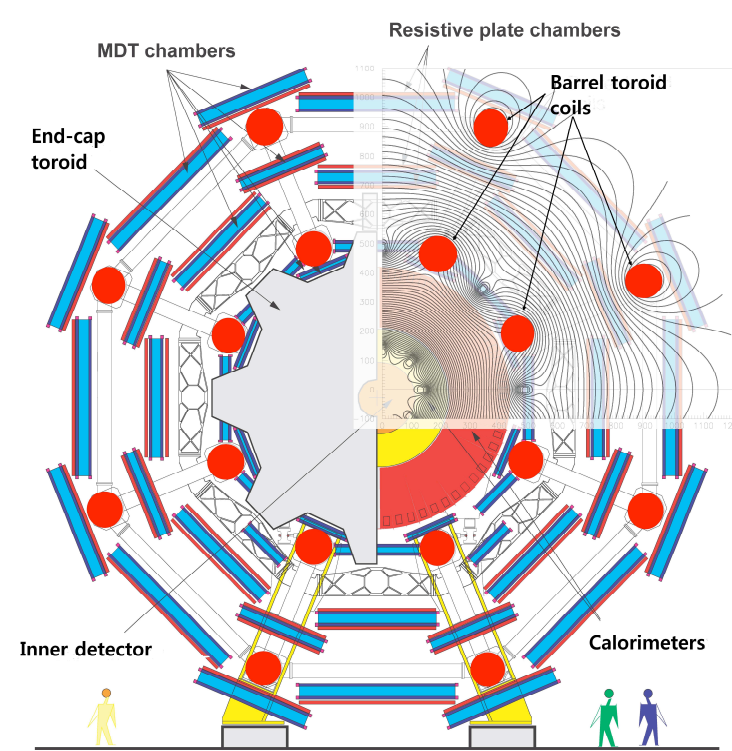

Fig. 1. Cross-section of the ATLAS Muon Barrel spectrometer, showing the three layers of Muon chambers, the Barrel toroid with coils below the outer chamber layer, and the Endcap toroid

This is an Open Access article distributed under the terms of the Creative Commons Attribution License 2.0, which permits unrestricted use, distribution, and reproduction in any medium, provided the original work is properly cited. 
sketch of the ATLAS Muon Barrel cross-section, where the three layers of Muon chambers are visible in blue.

They are immersed in a magnetic field, generated by a toroidal superconducting magnet composed of 8 air coils placed between the outer and inner layers of detectors. In the right-top quadrant, the magnetic field lines are overlapped to the spectrometer with a pitch of $0.1 \mathrm{~T}$. The average $B$ value around the chambers is of the order of 1 T. Because of this extremely high magnetic field, no power supply for the chamber front-end electronics can be placed on-detector, as it would be desirable [5].

For such a reason, power supplies of the Muon spectrometer are placed in crates allocated inside racks on the balconies on the sides of the experiment, at about 20 $\mathrm{m}$ distance from chambers. This solution requires a very large amount of cables to transport power to the chamber front-end electronics, as illustrated in figure 2.

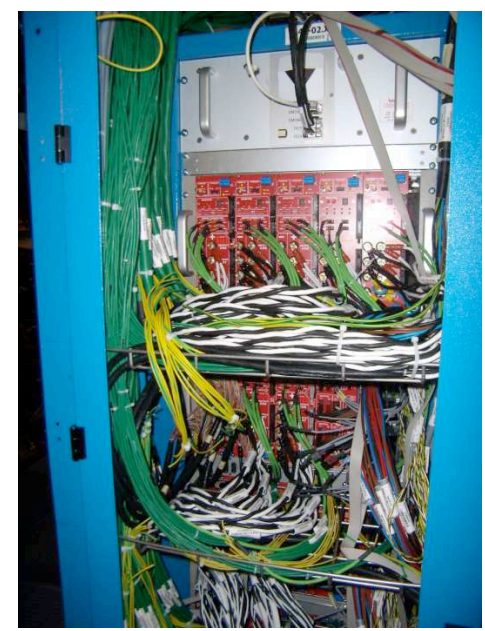

Fig. 2. Picture of an ATLAS Muon power supply rack. The green cables are dedicated to the power delivery to chambers

The power loss of the full spectrometer due to the cables is about $4 \mathrm{~kW}$, out of the $33 \mathrm{~kW}$ delivered (12\%) [6].

Another constraint, seriously limiting the on-chamber placement of the power supplies, is the very hostile radiation environment in their proximity, which is of the order of $10^{3}$ Gy of gammas, $10^{12} / \mathrm{cm}^{2}$ of $1 \mathrm{MeV}$ equivalent neutrons and $10^{11} / \mathrm{cm}^{2}$ of $20 \mathrm{MeV}$ hadrons, integrated over 10 years of activity [4].

After the so-called "Phase 2" upgrade of the LHC, foreseen in 2020 - 2022, the magnetic field will not be modified, while radiation values will be magnified by a factor of 5. The possibility of placing power supplies directly on-chamber will allow the saving of a lot of power, clearly related to the final cost of the power system.

At present, several investigations are looking for power devices working in such a high radiation area, and some are already identified [7], while the only solution for getting a DC-DC power supply converter working in a very high magnetic field seems to be the use of coreless inductors.

This article underlines a new approach to this problem, describing the development of the fabrication process of inductor cores made of a special material able to cope with the above mentioned magnetic field [8].

\section{Experimental}

In order to satisfy the high performances requested by the project for use in switching applications (high saturation induction and low coercive field) the research was focused on FeSi alloys $[9,10]$. The $\mathrm{Si}$ presence in the mixtures introduces, from the point of view of electrical properties, a better inductor core performance, due to the reduction of induced current loops in the material under workload and, from the mechanical one, the avoidance of magnetostriction phenomena [11]. Moreover, the $\mathrm{Si}$ presence decreases anisotropy thus enhancing soft magnetic properties [12].

\subsection{Starting materials}

Two kinds of iron-silicon powder were investigated. The first one was a pre-alloyed FeSi powder manufactured by Höganäs Belgium S.A, FESI $68 \mathrm{HQ}$, that shows a very fine granulometry distribution $(10-45 \mu \mathrm{m})$ and a near spherical morphology as shown in figure 3 .

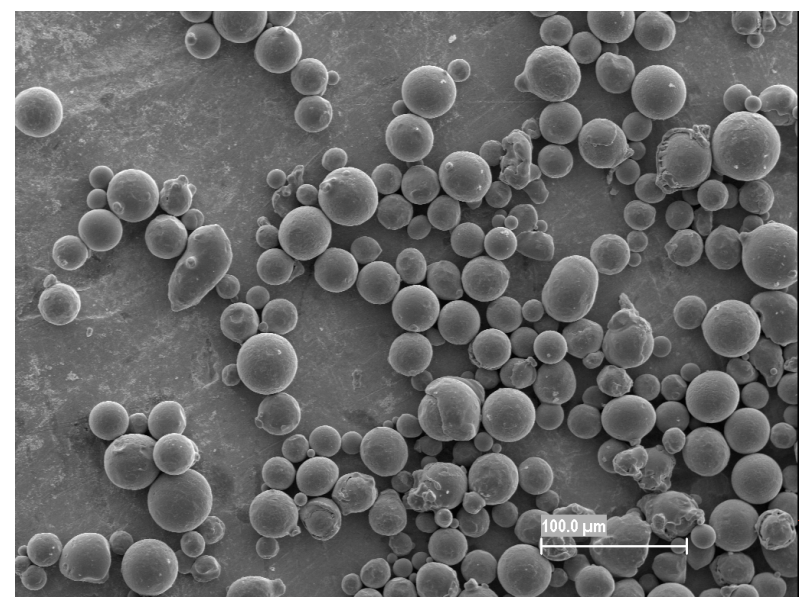

Fig. 3. Scanning Electron Microscopy (SEM) image of FESI 68 HQ powder morphology

The second one was a mixture of Fe NV powder (supplied by Metalpolveri SrL, Italy) and Si SIL A10 powder (manufactured by H.C. Stark, Germany), mixed in the same percentage of the pre-alloyed powder (i.e. Si amount around $6.5-6.8 \%$ ). Figure 4 shows the SEM morphology of Fe NV powder.

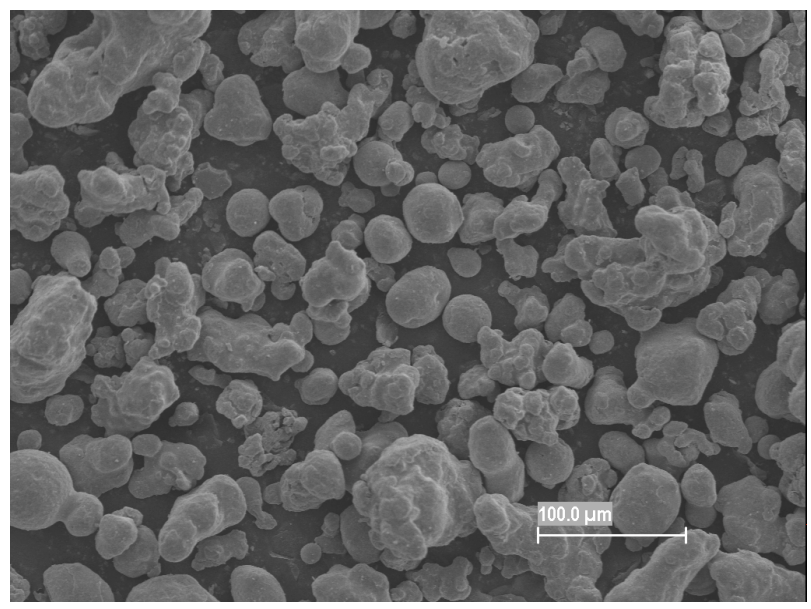

Fig. 4. SEM image of Fe NV powder morphology 
In comparison with FESI $68 \mathrm{HQ}$, Fe NV powder shows a more irregular shape and a lightly greater average grain size; the SIL A10 powder is submicronical and irregular in shape too. The mixture was prepared in a turbo-mixer. On all the powders an Energy Dispersive Spectroscopy (EDS) was performed in order to inspect their qualitative composition and purity.

\subsection{Process development}

Due to the component final shape and to the advantages of Metal Injection Moulding (MIM), such as the possibility to get complex shapes and large scale production, with reference to conventional production routes, MIM was chosen by FN to develop the magnetic cores.

The first step of the process consists in the choice of the binder system (based on thermo-polymers and waxes) to be mixed with the powder in order to allow the injection moulding of the powder itself into a proper mould. The binder system, in terms of kind and quantity, is also fundamental for the subsequent phases because, if it burns out in a not correct way during debinding, the component will show defects that are detrimental for its final integrity after sintering. At FN plant six different binder systems were experimented with a constant powder loading ( $90 \mathrm{wt} . \%$ of metal powder). Starting from the two metallic powders (the FeSi $68 \mathrm{HQ}$ and the in house $\mathrm{Fe}+\mathrm{Si}$ mixture) combined with these six binder systems, twelve kinds of feedstock (that is a pelletized mixture of metal powder and thermoplastic binder made by extrusion) were produced and tested.

The twelve kinds of feedstock were tested in an injection moulding machine (Negri e Bossi NB 100) equipped with a mould designed and realised on purpose in order to get specimens (a ring and a parallelepiped bar) according to the IEC 60404-04 standard for magnetic characterization.

The mould has a cavity and consists of a pathway for filling the cavity with ejectors for extracting the component from the cavity. The flow and thermal behaviour of the MIM feedstock are sufficiently different from conventional thermoplastics that special effort is needed to ensure uniform mould filling.

For injection moulding plastic components and filled polymers, the cavity dimensions are close to those of the final component, in contrast the cavity dimensions for MIM are oversized to accommodate subsequent sintering shrinkage. Another difference is the mould temperature: in MIM the mould temperature must be controlled to ensure final component dimensional control. To get the best density value in the injected component, called "green" due to the presence of the binder, the proper process conditions (temperature of the material, injection speed and screw feeding speed, injection time, hold pressure time, cooling time) were studied and implemented for each kind of feedstock. The specimens show a "green" density of about 55\% theoretical density of the metallic powder.

\subsection{Debinding and sintering}

In order to flush out the plastic binder it's necessary a debinding phase. Thermogravimetric analysis and differential scanning calorimetry (TGA/DSC) were performed, using Mettler-Toledo DSC1, on the different feedstocks to study how the thermoplastic binder flushes out during the thermal cycle. In figure 5 a TGA diagram, related to one kind of feedstock, is shown.

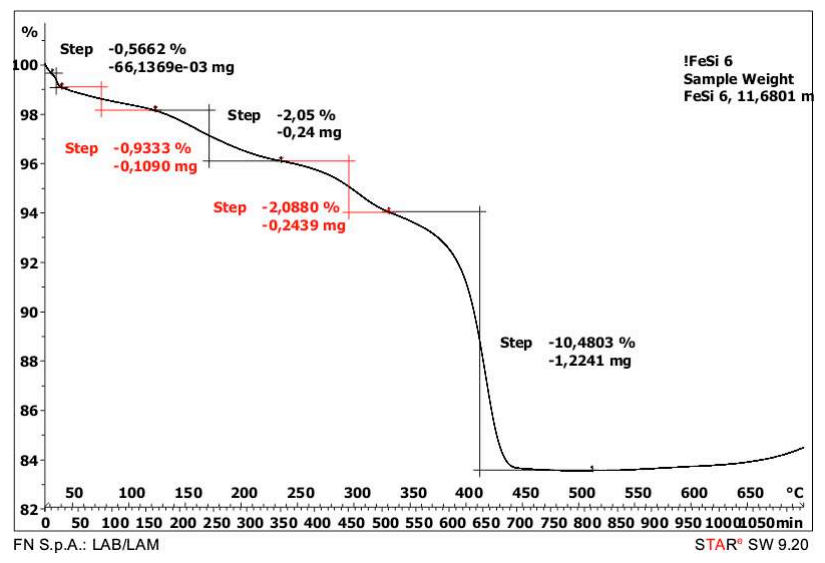

Fig. 5. TGA diagram relative to feedstock FeSi 6

The debinding step goes to $600{ }^{\circ} \mathrm{C}$, while the sintering phase reaches a temperature of $1260{ }^{\circ} \mathrm{C}$. In order to avoid defects and gaps the debinding needs to be slow enough, while, to achieve the best magnetic characteristics, the use of hydrogen atmosphere is recommended. Different thermal cycles were experimented, changing the temperature rate and the atmosphere. Differences were found between the two powders under study. The debinding and sintering process optimization is in progress. In figure 6 the change in dimensions from the "green" status to the sintering one, due to the sintering shrinkage, is shown.

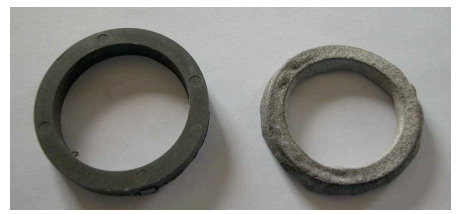

Fig. 6. Dimension changes before and after sintering

\subsection{Preliminary characterisation}

A preliminary characterisation was done. In particular, the samples were controlled "as green" in order to measure the "green" density and their homogeneity was investigated by SEM.

A few samples were exposed to $\gamma$ rays irradiation at ENEA Casaccia Res. Centre, Rome, Italy in the ${ }^{60} \mathrm{Co}$ radioisotope "Calliope" source. Test have been carried on at dose rate conditions of $100 \mathrm{~Gy} / \mathrm{h}$ during two days of exposure in the 24 hours. The dose of $5 \mathrm{kGy}$ is enough to model, in first approximation, the absorbed dose in particle collider experiments. Before and after $\gamma$ exposure the specimens were analysed by FT-IR (Fourier Transform - Infra Red) vibrational spectroscopy, by SEM and their magnetic characteristics were investigated: no appreciable effects were noted, apart a local initial fusion of the polymeric component documented by SEM [13]. In the next months, a qualification of the developed sintered soft materials under $\gamma$ rays irradiation is foreseen. 
Concerning the sintered samples, SEM and EDS analyses were carried out in order to investigate the effect of the sintering atmosphere on the material. The differences in grain size and morphology are typical.

With reference to the magnetic characterisation, made on torus shaped specimens using the system sketched in figure 7, as the sintered components don't show the specification density yet because the optimization of the process is still in progress, the magnetic characteristics don't comply yet with the specification in terms of B, even if interesting. In Fig. 8 and 9 the isteresys loop and the B-H curve are shown.

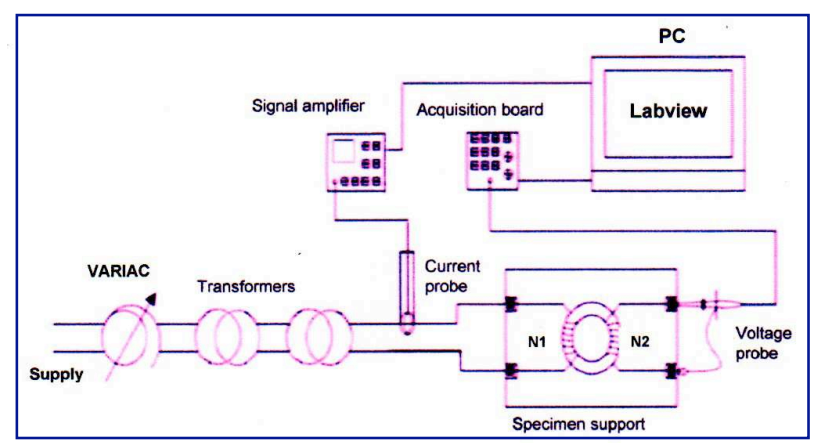

Fig. 7. Sketch of the system used for preliminary magnetic characterisation (courtesy of Turin Polytechnic)

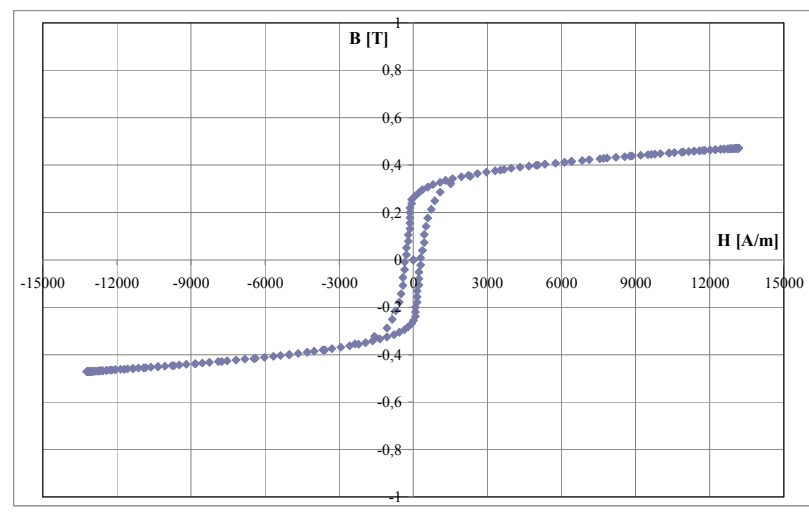

Fig. 8. Isteresys loop

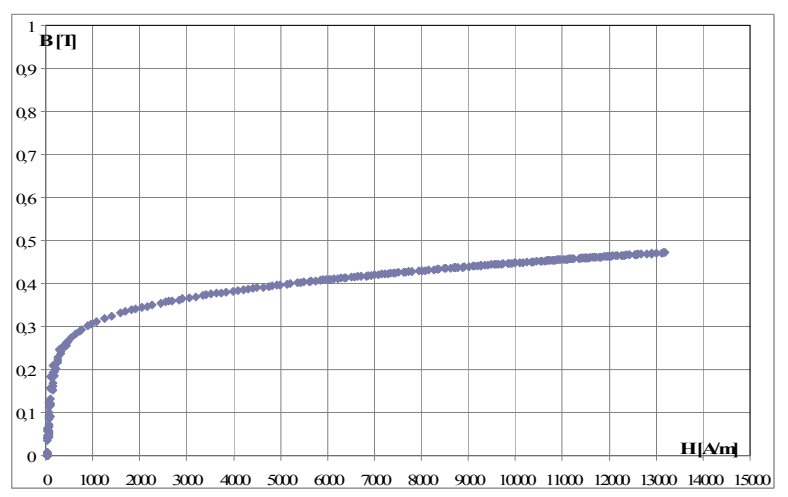

Fig. 9. B-H curve

\section{Conclusions}

In this text the description of the activities carried on at FN plant to develop high characteristic inductor cores to be used in extreme conditions of irradiation and high external magnetic fields was shown.
In order to fulfil the requested characteristics, two powders, in the FeSi alloys material field, were chosen; one is a commercial pre-alloyed powder (FESI $68 \mathrm{HQ}$ Höganäs), the other one consists in an house made mixture of two commercial powders (Fe NV Metalpolveri and Si SIL A10 H.C. Starck).

Due to the final complex shape of the components and to the proper advantages of the technique, Metal Injection Moulding (MIM) was chosen to produce the prototypes.

With the two starting materials and six binder combinations, twelve kinds of feedstock were developed and tested using an injection moulding machine equipped with a mould designed and realised on purpose. The "green" samples were characterised and TGA curves were made in order to define the debinding cycle. The optimization of this phase, together with the sintering to $1260^{\circ} \mathrm{C}$ in reducing atmosphere, is still in progress.

The preliminary microstructural characterisation of the first sintered prototypes suggested modifications to the process for the component optimization.

The preliminary magnetic characterisation showed interesting features, but it's necessary to optimize the process in order to get the specification values.

\section{Acknowledgments}

The authors would like to acknowledge INFN for financing the research activity inside the Apollo experiment.

A special thank goes to all FN SpA laboratory staff, in particular to Dr. Loredana Mannarino for SEM analyses, Dr. Valentina Naticchioni for TGA test, Mr. Maurizio Timo for technical support and to Pro.Ba. srl for the sample sintering in $\mathrm{H}_{2}$ atmosphere, to Eng. Emir Poskovic (Turin Polytechnic - Alessandria site) for magnetic test and to Dr. Alessia Cemmi (ENEA) for FTIR analyses.

\section{References}

1. G. Aad et al., J. of Instr. 3 S08003 (2008)

2. A. Loginov et al., ATL-UPGRADE-PROC-2011-002

3. V. Hedberg and M. Shupe, ATL-TECH-2004-002

4. The ATLAS Muon Collaboration, The ATLAS Muon Spectrometer technical design report. CERNLHCC/97-22, ISBN 92-9083-108-1 (1997)

5. M. Beretta et al., IEEE Trans. on Nucl. Sc. 51-5 (2004)

6. Y. Arai et al., J. of Instr. 3 P09001 (2008)

7. See for example presentations at TWEPP workshop: http://twepp11.hephy.at/home/previous-workshops/

8. M. Alderighi et al., J.of Instr.7 C03012 (2012)

9. H. Gravila and V. Ionita, J. of Optoel. and Adv. Mat. 4-2 (2002)

10. D. Ruitz et al., Magnetics, IEEE Trans. 41, 3286 (2005)

11. M. Takahashi et al., Magnetics, IEEE Trans. 23, 3068 (2007)

12. J. Diaz et al., Magnetics, IEEE Trans. 38, 2811 (2002)

13. S. Baccaro et al., Radiation damage in soft ferromagnetic materials for enhanced inductor cores operating in extreme environments, Proc. of $13^{\text {th }}$ ICATTP Conference, Como (2011) 